\title{
Comparative analysis of three availability systems with warm standby components, fault detection delay and general repair times
}

\author{
Tseng-Chang Yen ${ }^{\mathrm{a}}$, Kuo-Hsiung Wang ${ }^{\mathrm{b},{ }^{*}, \text { Wu-Lin Chen }}{ }^{\mathrm{b}}$ \\ ${ }^{\mathrm{a}}$ Department of Applied Mathematics, National Chung-Hsing University, Taichung 402, Taiwan \\ ${ }^{\mathrm{b}}$ Department of Computer Science and Information Management, Providence University, Taichung 43301, Taiwan \\ *Corresponding Author: khwang@pu.edu.tw
}

\begin{abstract}
We deal with three availability systems with warm standby components, fault detection delay and general repair times. Failure times and repair times of failed components are exponentially and generally distributed, respectively. Detection delay times are also exponentially distributed. A recursive method is used to develop steady-state availability, ( $A v)$, for three systems. We present extensive numerical computations to compare three systems for the $A v$ based on specific values given to the system parameters.
\end{abstract}

Keywords: Availability, comparative analysis, detection delay, supplementary variable, warm standby components.

\section{Introduction}

We study three availability systems with warm standby components, detection delay and general repair times. A recursive method is used to develop steady-state availability, $A v$, for three systems. The concept of detection delay for a repairable system was introduced by $\operatorname{Trivedi}^{(1)}$. Wang and Pearn ${ }^{(2)}$ studied the cost benefit analysis of series systems with warm standby components. They presented the explicit expressions for the mean time to failure and the steady-state availability for three configurations. Cost benefit analysis of availability systems with warm standby units and imperfect coverage was investigated by Wang and $\mathrm{Chiu}^{(3)}$. Further, they explored a comparative analysis which provided numerical results to show the effects of various values of system parameters to the cost/benefit ratios. Recently, Wang and $\mathrm{Chen}^{(4)}$ considered the availability characteristics between three different systems with reboot delay, switching failures, and general repair times. The reliability characteristics of a repairable system with balking and reneging was examined by Ke and Wang ${ }^{(5)}$. A repairable system with imperfect coverage and reboot was analyzed by $\mathrm{Ke}$ and $\mathrm{Liu}^{(6)}$. They derived the steady-state probabilities of down units at an arbitrary epoch. Singh et al. ${ }^{(7)}$ developed the transitional state probabilities, asymptotic behavior and some system characteristics such as reliability, availability, mean time to system failure, and the cost effectiveness of the system using the supplementary variable technique.

$\mathrm{Ke}$ et al $^{(8)}$ considered a repairable system with detection, imperfect coverage and reboot by means of the Bayesian approach. An application of stochastic Petri nets to compute the availability of safety critical on demand systems was proposed by Kleyner and Volovoi ${ }^{(9)}$. Cekyay, and Ozekici ${ }^{(10)}$ derived system reliability, mean time to failure, and steady-state availability on coherent systems and series connection of $k$-out-of- $n$ standby subsystems with exponentially distributed component lifetimes. A constructive method to optimize the availability of a system through modeling the dependency of the components was proposed by Yu et al. ${ }^{(11)}$.

\section{System description}

For the sake of discussion, we consider a data center requires a 30MW power electricity, and assume that the electricity generation capacity of components is available in units of 30MW, 15MW, and 10MW. To provide reliable and stable power supply, there are standby components, and all primary and standby components are continuously monitored by a fault detecting device to identify if they fail. The standby components are always required for the failure situation. Primary and standby components are assumed to be repairable. The primary and warm standby components fail independently and their times to failure are 
exponentially distributed with parameters $\lambda$ and $\alpha$ $(0<\alpha<1)$, respectively. When one of primary components fails, an available warm standby with failure rate $\alpha$ is converted into a primary component with failure rate $\lambda$. Whenever one of these components fails, it immediately detect other component. We assume that the detection delay has an exponential distribution with parameter $\delta$. During the time the fault is being detected, and the last primary component fail, the system is assumed to fail. Such a fault is called a near-coincident fault. The system fails when the standby components are emptied for which we defined as the state of system failure $(s f)$. It is assumed that the time-to-repair of the components are independent and identically distributed (i.i.d.) random variables having a distribution $B(u)(u \geq 0)$, a probability density function $b(u)(u \geq 0)$ and mean repair time $b_{1}$. If the remaining electricity generation capacity is less than $30 \mathrm{MW}$, the system will stop working. When the failed component arrives at the repair facility, if the server is available, the component will be repaired. If a primary component (or a standby component) fails, it is immediately repaired based on the first-come, first-served (FCFS) discipline. We assume that the server can repair only one failed component at a time, and that the service is independent of the failure of components. Once a component is repaired, it is as good as new.

We consider the following three availability systems: the first system consists of one $30 \mathrm{MW}$ primary component and one $30 \mathrm{MW}$ warm standby component. The second system is composed of two $15 \mathrm{MW}$ primary components and one $15 \mathrm{MW}$ warm standby component. The third system includes three $10 \mathrm{MW}$ primary components and two $10 \mathrm{MW}$ warm standby components.

\subsection{Availability system 1}

Availability system 1 consists of one 30 MW primary component and one $30 \mathrm{MW}$ warm standby component. The state-transition-rate diagram of availability system 1 is shown in Fig. 1, where the state labeled $(1,1)$ denotes the detection stage. The transition from state $(1,1)$ to state $(1,0)$ (at rate $\delta$ ) indicates the detection of the fault.

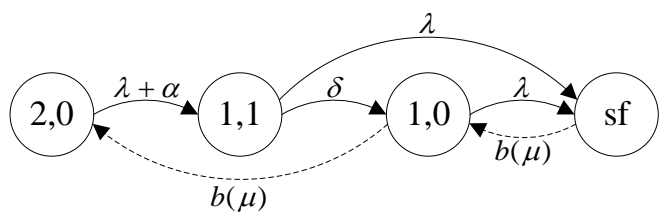

Fig. 1. The state-transition-rate diagram of availability system 1 .
The state labeled $s f$ denotes the system failure. We define

$$
P_{1,1}(u)=b(u) P_{1,1} .
$$

then we obtain the following steady-state equations

$$
\begin{gathered}
0=-(\lambda+\alpha) P_{2,0}+P_{1,0}(0), \\
0=-(\lambda+\delta) P_{1,1}+(\lambda+\alpha) P_{2,0}, \\
-\frac{d}{d u} P_{1,0}(u)=-\lambda P_{1,0}(u)+\delta b(u) P_{1,1}+b(u) P_{s f}(0), \\
-\frac{d}{d u} P_{s f}(u)=\lambda P_{1,0}(u)+\lambda b(u) P_{1,1} .
\end{gathered}
$$

We assume that the detection state $(1,1)$ is a system down state. For availability system 1, the explicit expression for the steady-state availability, $A v_{l}$, is given by

$$
A v_{1}=P_{2,0}+P_{1,0}=\frac{(\lambda+\delta)\left[\lambda+\alpha-\alpha B^{*}(\lambda)\right]}{\lambda \Delta_{1}}
$$

Special cases

We develop the following explicit expressions for the $A v_{1 M}, A v_{1 E}, A v_{1 D}$ for three different repair time distributions such as exponential, $k$-stage Erlang, and deterministic, respectively.

Case 1. Exponential repair time

The explicit expression for the $A v_{1 M}$ is given by

$$
A v_{1 M}=\frac{(\lambda+\delta)[(\lambda+\alpha)(\mu+\lambda)-\alpha \mu]}{\lambda(\mu+\lambda) \Delta_{1}} .
$$

Case 2. $k$-stage Erlang repair time

The explicit expression for the $A v_{1 E}$

$$
A v_{1 E}=\frac{(\lambda+\delta)\left[(\lambda+\alpha)(k \mu+\lambda)^{k}-\alpha(k \mu)^{k}\right]}{\lambda(k \mu+\lambda)^{k} \Delta_{1}} .
$$

Case 3. Deterministic repair time

The explicit expression for the $A v_{1 D}$ is given by

$$
A v_{1 D}=\frac{(\lambda+\delta)\left(\lambda+\alpha-\alpha e^{-\lambda / \mu}\right)}{\lambda \Delta_{1}} .
$$

\subsection{Availability system 2}

Availability system 2 consists of two $15 \mathrm{MW}$ primary 
components and one $15 \mathrm{MW}$ warm standby component. The state-transition-rate diagram of availability system 2 is presented in Fig. 2, where the state labeled $(2,1)$ represents the detection stage.

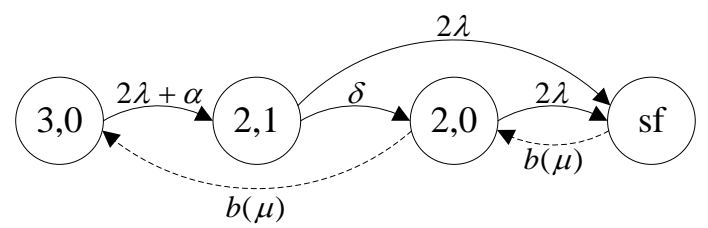

Fig. 2. The state-transition-rate diagram of availability system 2 .

We get the following steady-state equations:

$$
\begin{gathered}
0=-(2 \lambda+\alpha) P_{3,0}+P_{2,0}(0) \\
0=-(2 \lambda+\delta) P_{2,1}+(2 \lambda+\alpha) P_{3,0} \\
-\frac{d}{d u} P_{2,0}(u)=-2 \lambda P_{2,0}(u)+\delta b(u) P_{2,1}+b(u) P_{s f}(0) \\
-\frac{d}{d u} P_{s f}(u)=2 \lambda P_{2,0}(u)+2 \lambda b(u) P_{2,1}
\end{gathered}
$$

For availability system 2, the explicit expression for the steady-state availability, $A v_{2}$, is given by

$$
A v_{2}=P_{3,0}+P_{2,0}=\frac{(2 \lambda+\delta)\left[2 \lambda+\alpha-\alpha B^{*}(2 \lambda)\right]}{2 \lambda \Delta_{2}} .
$$

Special cases

For availability system 2 , we get

$$
\begin{aligned}
& A v_{2 M}=\frac{(2 \lambda+\delta)[(2 \lambda+\alpha)(\mu+2 \lambda)-\alpha \mu]}{2 \lambda(\mu+2 \lambda) \Delta_{2}}, \\
& A v_{2 E}=\frac{(2 \lambda+\delta)\left[(2 \lambda+\alpha)(k \mu+2 \lambda)^{k}-\alpha(k \mu)^{k}\right]}{2 \lambda(k \mu+2 \lambda)^{k} \Delta_{2}}, \\
& A v_{2 D}=\frac{(2 \lambda+\delta)\left(2 \lambda+\alpha-\alpha e^{-2 \lambda / \mu}\right)}{2 \lambda \Delta_{2}} .
\end{aligned}
$$

\subsection{Availability system 3}

Availability system 3 includes three $10 \mathrm{MW}$ primary components and two $10 \mathrm{MW}$ warm standby components. The state-transition-rate diagram of availability system 3 is shown in Fig. 3, the states labeled $(4,1),(3,2)$ and $(3,1)$ represent the detection stages.

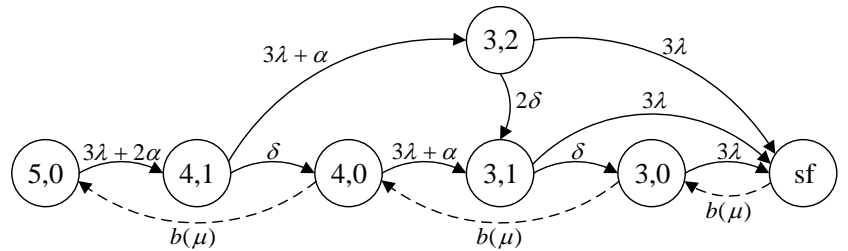

Fig. 3. The state-transition-rate diagram of availability system 3 .

Using the same procedure, then we get the steady-state equations. For availability system 3 , the explicit expression for the steady-state availability, $A v_{3}$, is given by

$$
\begin{aligned}
& A v_{3}=\left\{1+\frac{(3 \lambda+2 \alpha)\left[1-B^{*}(3 \lambda+\alpha)\right]}{(3 \lambda+\alpha) B^{*}(3 \lambda+\alpha)}\right. \\
& \left.+\frac{\frac{(3 \lambda+2 \alpha)\left[3 \lambda+\alpha+\delta-\delta B^{*}(3 \lambda+\alpha)\right]}{(3 \lambda+\alpha+\delta) B^{*}(3 \lambda+\alpha)}\left[1-B^{*}(3 \lambda)\right]}{3 \lambda B^{*}(3 \lambda)}\right\} P_{5,0} .
\end{aligned}
$$

\section{Special cases}

For availability system 3, we obtain the following expressions for the $A v_{3 M}, A v_{3 E}, A v_{3 D}$ :

$$
\begin{aligned}
A v_{3 M}=\{ & (3 \lambda+2 \alpha)[(3 \lambda+\alpha+\delta)(\mu+3 \lambda+\alpha)-\delta \mu] \\
& +(3 \lambda+2 \alpha+\mu)(3 \lambda+\alpha+\delta)\} \frac{P_{5,0}}{\mu(3 \lambda+\alpha+\delta)},
\end{aligned}
$$

$$
\begin{aligned}
A v_{3 E}= & +\frac{(3 \lambda+2 \alpha)\left[(k \mu+3 \lambda+\alpha)^{k}-(k \mu)^{k}\right]}{(3 \lambda+\alpha)(k \mu)^{k}} \\
& +\frac{\Phi_{1}\left[(k \mu+3 \lambda)^{k}-(k \mu)^{k}\right]}{3 \lambda(k \mu)^{k}} P_{5,0},
\end{aligned}
$$

$$
\begin{aligned}
A v_{3 D}= & +\frac{(3 \lambda+2 \alpha)\left(e^{3 \lambda+\alpha / \mu}-1\right)}{3 \lambda+\alpha} \\
& +\frac{(3 \lambda+2 \alpha)\left[(3 \lambda+\alpha+\delta) e^{3 \lambda+\alpha / \mu}-\delta\right]\left(e^{3 \lambda / \mu}-1\right)}{3 \lambda+\alpha+\delta} P_{5,0},
\end{aligned}
$$

where

$$
\Phi_{1}=\frac{(3 \lambda+2 \alpha)\left[(3 \lambda+\alpha+\delta)(k \mu+\lambda)^{k}-\delta(k \mu)^{k}\right]}{(3 \lambda+\alpha+\delta)(k \mu)^{k}} .
$$




\section{Comparison of the three availability systems}

Click here and insert your conclusions text. We consider $\lambda=0.001, \alpha=0.0005, \quad \mu=0.1, \quad \delta=2.4$.

Comparison for the $A v_{i}$

We first consider the following three cases:

Case 1. We fix $\alpha=\lambda / 2, \mu=0.1, \delta=2.4$, and vary the values of $\lambda$ from 0.001 to 0.02 .

Case 2. We fix $\lambda=0.001, \alpha=\lambda / 2, \delta=2.4$, and vary the values of $\mu$ from 0.04 to 0.16 .

Case 3. We fix $\lambda=0.001, \alpha=\lambda / 2, \mu=0.1$, and vary the values of $\delta$ from 1 to 10 .

From Tables 1-3, numerical results can be found in analyzing the values of the $A v_{i M}, A v_{i E}, A v_{i D} \quad(i=1,2,3)$. We first note that the rank of three availability systems sorted by their steady-state availability does not depend on distributions of repair time but on the values of $\lambda, \mu$, and $\delta$. Next, we note that the steady-state availability of system 1 is larger than systems 2 and 3 for all cases.

Table 1. Comparison of the availability systems $1,2,3$ for $A v \cdot(\alpha=\lambda / 2, \mu=0.1, \delta=2.4)$

\begin{tabular}{ll}
\hline \multicolumn{1}{c}{ Range of $\lambda$} & \multicolumn{1}{c}{ Result } \\
\hline 1. Exponential & \\
$0.001<\lambda<0.001477$ & $A v_{1 M}>A v_{2 M}>A v_{3 M}$ \\
$0.001477<\lambda<0.013881$ & $A v_{1 M}>A v_{3 M}>A v_{2 M}$ \\
$0.013881<\lambda<0.02$ & $A v_{1 M}>A v_{2 M}>A v_{3 M}$ \\
2. 3-stage Erlang & \\
$0.001<\lambda<0.002459$ & $A v_{1 E}>A v_{2 E}>A v_{3 E}$ \\
$0.002459<\lambda<0.010156$ & $A v_{1 E}>A v_{3 E}>A v_{2 E}$ \\
$0.010156<\lambda<0.02$ & $A v_{1 E}>A v_{2 E}>A v_{3 E}$ \\
3. Deterministic & \\
$0.001<\lambda<0.003956$ & $A v_{1 D}>A v_{2 D}>A v_{3 D}$ \\
$0.003956<\lambda<0.007653$ & $A v_{1 D}>A v_{3 D}>A v_{2 D}$ \\
$0.007653<\lambda<0.02$ & $A v_{1 D}>A v_{2 D}>A v_{3 D}$ \\
\hline
\end{tabular}

Table 1. Comparison of the availability systems $1,2,3$

\begin{tabular}{cc}
\multicolumn{2}{c}{ for $A v \cdot(\lambda=0.001, \alpha=\lambda / 2, \delta=2.4)$} \\
\hline Range of $\mu$ & Result \\
\hline 1. Exponential & \\
$0.04<\mu<0.083678$ & $A v_{1 M}>A v_{3 M}>A v_{2 M}$ \\
$0.083678<\mu<0.16$ & $A v_{1 M}>A v_{2 M}>A v_{3 M}$ \\
2. 3-stage Erlang & \\
$0.04<\mu<0.067598$ & $A v_{1 E}>A v_{3 E}>A v_{2 E}$ \\
$0.067598<\mu<0.16$ & $A v_{1 E}>A v_{2 E}>A v_{3 E}$ \\
3. Deterministic & \\
$0.04<\mu<0.057929$ & $A v_{1 D}>A v_{3 D}>A v_{2 D}$ \\
$0.057929<\mu<0.16$ & $A v_{1 D}>A v_{2 D}>A v_{3 D}$ \\
\hline
\end{tabular}

Table 3. Comparison of the availability systems $1,2,3$ for $A v \cdot(\lambda=0.001, \alpha=\lambda / 2, \mu=0.1)$

\begin{tabular}{ll}
\hline \multicolumn{1}{c}{ Range of $\delta$} & \multicolumn{1}{c}{ Result } \\
\hline 1. Exponential & \\
$1<\delta<3.350022$ & $A v_{1 M}>A v_{2 M}>A v_{3 M}$ \\
$3.350022<\delta<10$ & $A v_{1 M}>A v_{3 M}>A v_{2 M}$ \\
2. 3-stage Erlang & \\
$1<\delta<4.980970$ & $A v_{1 E}>A v_{2 E}>A v_{3 E}$ \\
$4.980970<\delta<10$ & $A v_{1 E}>A v_{3 E}>A v_{2 E}$ \\
3. Deterministic & \\
$1<\delta<6.611954$ & $A v_{1 D}>A v_{2 D}>A v_{3 D}$ \\
$6.611954<\delta<10$ & $A v_{1 D}>A v_{3 D}>A v_{2 D}$ \\
\hline
\end{tabular}

\section{Conclusions}

In this paper, we first derive the steady-state availability $A v$ of three availability systems with warm standby components, fault detection delay and general repair times. Nest, for each availability system, the explicit expressions for the $A v$ for three different repair time distributions, such as exponential, 3-stage Erlang, and deterministic are developed. Finally, we rank three availability systems based on the $A v_{i}$ for three various repair time distributions. 


\section{References}

(1) Trivedi, KS: "Probability and Statistics with Reliability, Queueing and Computer Science Applications", John Wiley and Sons, New York, 2002.

(2) Wang KH, Pearn WL: "Cost benefit analysis of series systems with warm standby components", Mathematics Method of Operations Research, Vol. 58, pp. 247-258, 2003

(3) Wang KH, Chiu LW: "Cost benefit analysis of availability systems with warm standby units and imperfect coverage", Applied Mathematics and Computations, Vol. 172, pp. 1239-1256, 2006

(4) Wang KH, Chen YJ.: "Comparative analysis of availability between three systems with general repair times, reboot delay and switching failures", Applied Mathematics and Computations, Vol. 215, pp. 384-94, 2009

(5) Ke JC, Wang KH: "The reliability analysis of balking and reneging in a repairable system", Quality and Reliability Engineering International, Vol. 18, pp. 467-478, 2002

(6) Ke JC, Liu TH: "A repairable system with imperfect coverage and reboot", Applied Mathematics and Computation, Vol. 246, pp. 148-58, 2014

(7) Singh VV, Singh SB, Ram M, Goel CK: "Availability, MTTF and cost analysis of a system having two units in series configuration with controller", International Journal of System Assurance Engineering and Management, Vol. 4, pp. 341-52, 2013

(8) Ke JC, Lee SL, Hsu YL: "On a repairable system with detection, imperfect coverage and reboot: Bayesian approach", Simulation Modelling Practice and Theory, Vol. 16, pp. 353-67, 2008

(9) Kleyner A, Volovoi V: "Application of Petri nets to reliability prediction of occupant safety systems with partial detection and repair", Reliability Engineering and System Safety, Vol. 95, pp. 606-13, 2010

(10) Cekyay B, Ozekici S: "Reliability, MTTF, and steady-state-state availability analysis of systems with exponential lifetimes", Applied Mathematical Modeling, Vol. 39, pp. 284-296, 2015

(11) Yu H, Chu C, Chatelet E: "Availability optimization of a redundant system through dependency modeling", Applied Mathematical Modeling, Vol. 38, pp. 4574-85, 2014 